\title{
MisCELLANEOUS
}

\section{Thermography in ocular inflammation}

\author{
Ankush A Kawali \\ Uvea Clinic, Aravind Eye Hospital, Avinashi Road, Coimbatore, Tamil Nadu, India
}

Correspondence: Dr. Ankush Ashok Kawali, Uveitis and Ocular Immunology Clinic, Narayana Nethralaya, Chord Road, Rajaji Nagar, Bangalore, India. E-mail: akawali332@gmail.com

\begin{abstract}
Background and Objectives: The purpose of this study was to evaluate ocular inflammatory and non-inflammatory conditions using commercially available thermal camera. Materials and Methods: A non-contact thermographic camera (FLIR P 620) was used to take thermal pictures of seven cases of ocular inflammation, two cases of non-inflammatory ocular pathology, and one healthy subject with mild refractive error only. Ocular inflammatory cases included five cases of scleritis, one case of postoperative anterior uveitis, and a case of meibomian gland dysfunction with keratitis (MGD-keratitis). Non-inflammatory conditions included a case of conjunctival benign reactive lymphoid hyperplasia (BRLH) and a case of central serous chorio-retinopathy. Thermal and non-thermal photographs were taken, and using analyzing software, the ocular surface temperature was calculated. Results: Patient with fresh episode of scleritis revealed high temperature. Eyes with MGD-keratitis depicted lower temperature in clinically more affected eye. Conjunctival BRLH showed a cold lesion on thermography at the site of involvement, in contrast to cases of scleritis with similar clinical presentation. Conclusion: Ocular thermal imaging is an underutilized diagnostic tool which can be used to distinguish inflammatory ocular conditions from non-inflammatory conditions. It can also be utilized in the evaluation of tear film in dry eye syndrome. Its applications should be further explored in uveitis and other ocular disorders. Dedicated "ocular thermographic" camera is today's need of the hour.
\end{abstract}

Key words: Dry eye; inflammation; scleritis; thermography; uveitis

\section{Introduction}

Thermography is not a new word in today's world. It has been used primarily by military, construction workers, fire fighters to see through smoke, and to spot heat leaks or the enemy.

Ocular thermography is still in its growing phase. There are few reports and small-scale studies done in various disciplines of ophthalmology. It has been used in the diagnosis of dry eye ${ }^{[1]}$ and to evaluate bleb functionality after glaucoma surgery. ${ }^{[2]}$ It has also been used to demonstrate low ocular temperature in vascular occlusions ${ }^{[3]}$ and high temperature in ocular inflammation. ${ }^{[4,5]}$ This is a report on

\begin{tabular}{|l|l|}
\hline \multicolumn{2}{|c|}{ Access this article online } \\
\hline Quick Response Code: & \\
\cline { 1 - 2 } & Website: \\
\hline & www.iji.org \\
\cline { 1 - 2 } & \\
\hline
\end{tabular}

the study of thermographic images of ocular inflammatory and non-inflammatory conditions.

\section{Materials and Methods}

A non-contact thermographic camera (FLIR P 620) was used to take thermal pictures of seven cases of ocular inflammation, two cases of non-inflammatory ocular pathology, and one healthy subject with mild refractive error only. Ocular inflammatory cases included five cases of scleritis (three anterior and two posterior), one case of postoperative anterior uveitis, and a case of bilateral meibomian gland dysfunction with keratitis (MGD-keratitis). Non-inflammatory conditions included a case of conjunctival benign reactive lymphoid hyperplasia (BRLH) and a case of central serous chorio-retinopathy (CSCR). Thermal and non-thermal photographs were taken using same magnification and same distance from the eye at atmospheric temperature of $27^{\circ} \mathrm{C}$, humidity $60 \%$, and with stable airflow. Participants were examined after being rested for $5 \mathrm{~min}$ in the examination room. Physiological blinking was allowed, no ocular medications were applied, and thermal barriers like eyeglasses or contact lenses were removed before photography. All images were transferred to a computer, and using analyzing software, 
average ocular surface temperature (OST) and temperature at the area of interest were calculated.

\section{Results}

The eye of the patient with a fresh episode of scleritis, which was not on treatment, showed hot colors on thermography [Figure 1] indicating higher temperature in right eye compared to fellow healthy eye. Eyes with scleritis on treatment showed slightly higher temperature compared to the fellow eye. A case of resolved necrotizing scleritis showed lower temperature at the site of the necrosis surrounded by normal tissue temperature [Figure 2]. Eye with postoperative anterior uveitis also revealed increased temperature, but paradoxically, eyes with MGD-keratitis depicted lower temperature in clinically more affected right eye (OD) [Figure 3]. Eye with conjunctival BRLH showed lower temperature at the site of the lesion [Figure 4]. Eyes with only refractive error but no other pathology showed no temperature difference between both the eyes. Figure 5 is the temperature scale ranged between $31.5^{\circ} \mathrm{C}$ to $38.5^{\circ} \mathrm{C}$.

\section{Discussion}

Ocular thermography is a relatively newer imaging modality. Attempts to calculate normal eye temperature

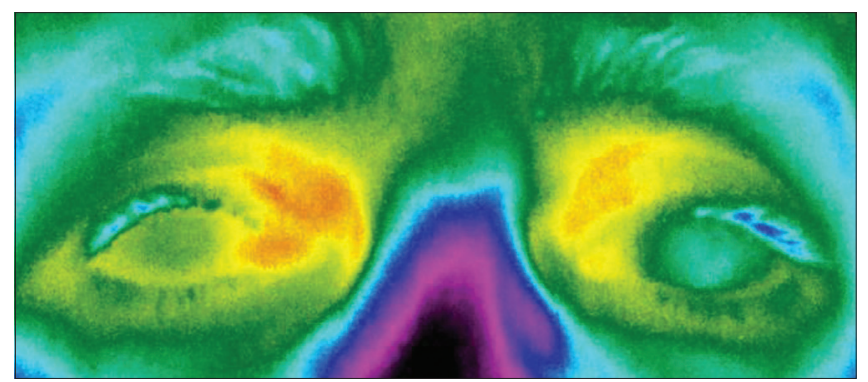

Figure 1: A 63-year-old female presented with OD scleritis. OD showed higher temperature compared to the fellow healthy eye

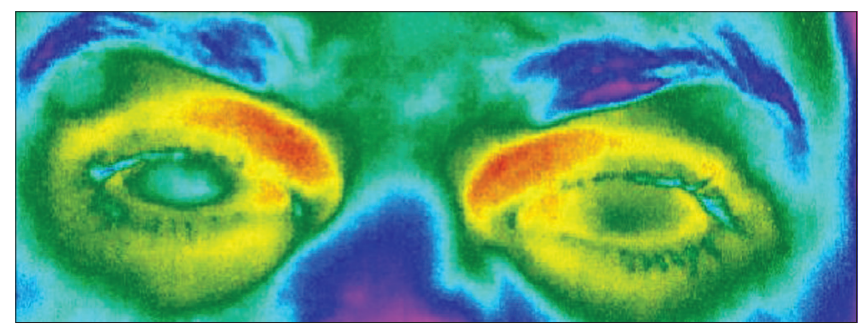

Figure 3: A 35-year-old male presented with bilateral MGD-keratitis. OD was affected more than OS. Paradoxically, OD shows low temperature as compared to OS were made way back in 1950s using contact thermometry. In 1968, R. Mapstone introduced infrared thermometry using bolometer, a non-contact method of ocular thermometry. ${ }^{[6]}$ The normal corneal temperature measured by thermography ranges from $35.4 \pm 0.1^{\circ} \mathrm{C}^{[5]}$ to $34.01 \pm 0.64^{\circ} \mathrm{C}^{[7]}$ and the mean inter-ocular difference of OST in normal individuals was found to be $0.21 \pm 0.18^{\circ} \mathrm{C} .^{[7]}$ Our first patient, a fresh case of scleritis who was not on any treatment, had higher OST difference $(\Delta t)$ between the two eyes as compared to the eyes on treatment for more than a week [Table 1]. This may suggest that ocular temperature rapidly decreases with anti-inflammatory treatment and has a positive correlation with the signs and symptoms. In this study, the case of MGD-keratitis (case 6) paradoxically showed low temperature in clinically more affected eye (OD). Rolando et al. also noted similar finding in dry eye and suggested that the ocular surface would be $0.10^{\circ} \mathrm{C}$ lesser in dry eyes due to the increased rate of evaporation. ${ }^{[8]}$ Hence, applying the same logic, it is clear that in case 6 in the present study, due to decreased tear film lipids owing to MGD, the worse

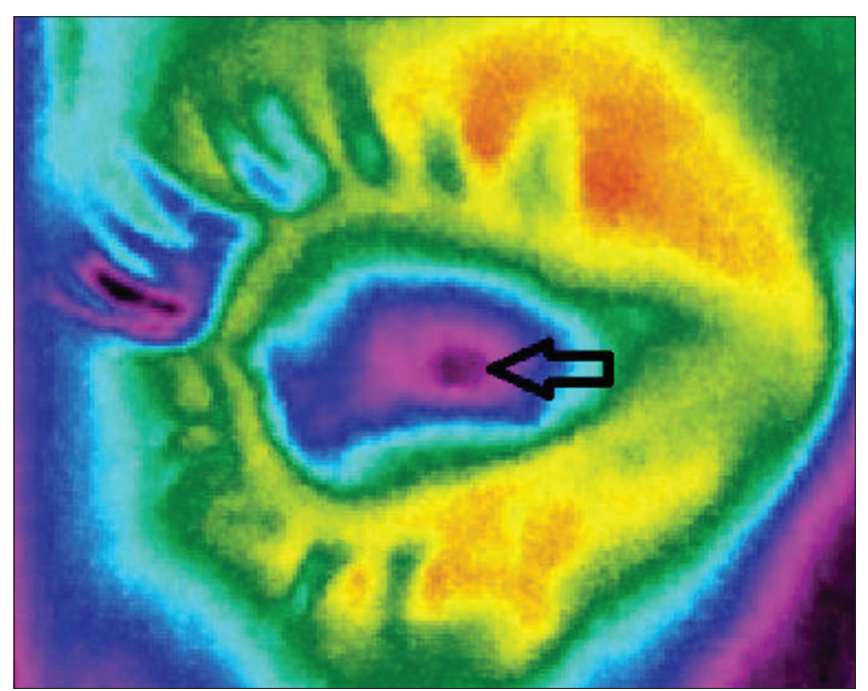

Figure 2: A 65-year-old female presented with necrotizing scleritis. Temperature at the site of the necrosis (arrow) was $31.8^{\circ} \mathrm{C}$

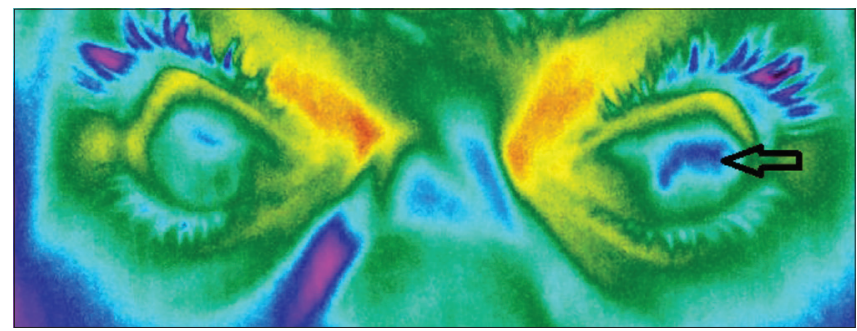

Figure 4: A 34-year-old male presented with conjunctival BRLH. Note low temperature at the site of the lesion (arrow)

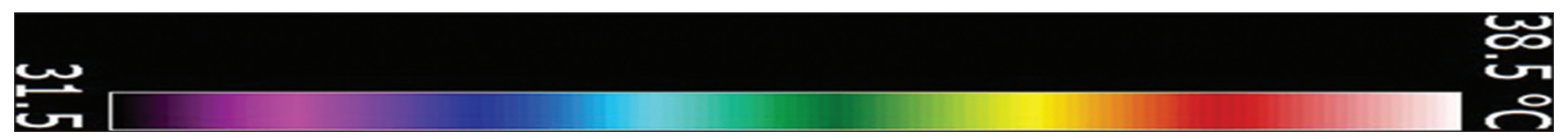

Figure 5: Thermal scale 
Table 1: Detailed patient information and temperature difference between affected and unaffected eye

\begin{tabular}{|c|c|c|c|c|c|c|}
\hline Case & Age/sex & Laterality & Diagnosis & Severity & On treatment for more than a week & $\Delta \mathrm{t}\left({ }^{\circ} \mathrm{C}\right)$ \\
\hline 1 & $63 / F$ & $\mathrm{RE}$ & Anterior scleritis & Moderate* & No & +1.125 \\
\hline 2 & $25 / \mathrm{M}$ & RE & Traumatic scleritis & Moderate* & Yes & +0.75 \\
\hline 3 & 18/M & $\mathrm{BE}$ & Posterior scleritis & $\begin{array}{l}\text { RE: Resolving } \\
\text { LE: Resolved }\end{array}$ & Yes & +0.22 \\
\hline 4 & $24 / F$ & RE & Posterior scleritis & Resolving & Yes & +0.125 \\
\hline 5 & $65 / F$ & RE & Necrotizing scleritis & Resolved & Yes & (one eyed) \\
\hline 6 & $35 / \mathrm{M}$ & $\mathrm{BE}(\mathrm{Rt}>\mathrm{Lt})$ & MGD-keratitis & $\begin{array}{l}\text { RE: Severe } \\
\text { LE: Mild }\end{array}$ & No & -0.625 \\
\hline 7 & $62 / \mathrm{M}$ & RE & Postop anterior uveitis & Moderate & Yes & +0.35 \\
\hline 8 & $32 / \mathrm{M}$ & LE & Conjunctival BRLH & $15 \times 10 \mathrm{~mm}$ mass & No & -0.2 \\
\hline 9 & 45/M & RE & CSCR & Shallow retinal detachment of 3 disc diameter & No & -0.175 \\
\hline 10 & $25 / F$ & $\mathrm{BE}$ & Only refractive error & - & No & 0 \\
\hline
\end{tabular}

$\Delta t\left({ }^{\circ} \mathrm{C}\right)$ : Difference of temperature between the affected eye and the other eye, ${ }^{*}$ Standardized grading for scleritis ${ }^{[9]}$, BRLH: Benign reactive lymphoid hyperplasia, RE: Right eye, LE: Left eye, BE: Both eyes, CSCR: Central serous chorio-retinopathy

eye (OD) was subjected to rapid evaporation of the tear film which resulted in a relatively colder eye on thermography.

Thermography can also be used to distinguish between a cold lesion of non-inflammatory condition from a hot lesion of ocular inflammation. Conjunctival BRLH is a poorly vascularized tumor, and hence, case 8 in the present study showed low temperature at the site of the lesion. In contrast case 1 who came with similar clinical presentation showed high temperature, suggestive of inflammation due to scleritis. Our cases of posterior scleritis had "minimal" increase in temperature as compared to the non-affected eye, as they were on treatment. This may suggest that posterior segment inflammation can also cause increase in OST, which can be picked up on thermography when posterior segment examination is difficult as in the case of mature cataract or when it is normal as in the case of mild posterior scleritis.

\section{Conclusion}

Ocular thermal imaging is an underutilized diagnostic tool which can be used to distinguish inflammatory ocular conditions from non-inflammatory conditions. It can also be utilized in the evaluation of tear film in dry eye syndrome and other corneal pathologies. Its applications should be further explored in uveitis and other ocular disorders. Dedicated "ocular thermographic" camera is today's need of the hour.

\section{Acknowledgment}

I sincerely thank to my colleagues Dr. Divya Sundaresh, Orbit clinic and Dr. Hardik Parikh, Cornea clinic of Aravind eye hospital, Coimbatore for contributing cases in this study. I also acknowledge the help of Mr. G. Suresh, PCI Ltd, Chennai for his excellent thermal photography and analysis.

\section{References}

1. Morgan PB, Tullo AB, Efron N. Infrared thermography of the tear film in dry eye. Eye 1995;9:615-8.

2. Kawasaki S, Mizoue S, Yamaguchi M, Shiraishi A, Zheng X, Hayashi $\mathrm{Y}$, et al. Evaluation of filtering bleb function by thermography. Br J Ophthalmol 2009;93:1331-6.

3. Mapstone R. Ocular thermography. Br J Ophthalmol 1970;54:751-4.

4. Mapstone R. Corneal thermal patterns in anterior uveitis. Br J Ophthalmol 1968;52:917-21.

5. Stefanie PetrouBinder. Thermography shows enormous promise for diagnosis and treatment of eye diseases. Eurotimes. Available from: http://www.escrs.org/eurotimes/march2003/thermo.asp [Last accessed on 2003 Mar].

6. Mapstone R. Determinants of corneal temperature. Br J Ophthalmol 1968;52:729-41.

7. Tan JH, Ng EY, Acharya UR. An efficient automated algorithm to detect ocular surface temperature on sequence of thermograms using snake and target tracing function. J Med Syst 2011;35:949-58.

8. Rolando M, Refojo MF, Kenyon KR. Increased tear evaporation in eyes with keratoconjunctivitis sicca. Arch Ophthalmol 1983;101:557-8.

9. Sen HN, Sangave AA, Goldstein DA, Suhler EB, Cunningham D, Vitale S, et al. A Standardized Grading System forscleritis. Ophthalmology 2011;118:768-71.

Cite this article as: Kawali AA. Thermography in ocular inflammation. Indian J Radiol Imaging 2013;23:281-3.

Source of Support: PCI, Itd, Chennai provided FLIR P620 thermal camera for this study, Conflict of Interest: None declared. 